\title{
Google trends as a surrogate marker of public awareness of diabetic retinopathy
}

\author{
Rajna Rasheed ${ }^{1} \cdot$ Sobha Sivaprasad ${ }^{2}$
}

Received: 10 February 2020 / Accepted: 16 March 2020 / Published online: 14 April 2020

(c) The Royal College of Ophthalmologists 2020

Diabetes and its complications are leading causes of morbidity and mortality globally. The global prevalence of diabetes is set to increase from the current figures of 463 million people to an estimated 700 million by 2045 [1]. Approximately 1:10 adults are already affected in some nations. Whilst new drugs, improved care pathways and increased allocation of national budgets for diabetes and its complications are most welcome, increasing patient and public awareness of this silent disease and its complications is also crucially important. A recent global survey by the International Diabetes Foundation showed that only one in two (50.1\%) people living with diabetes know that they have diabetes [1]. Traditionally, qualitative studies and questionnaire-based outcomes are used to provide most of the feedback on patient awareness. However, with advances in information technology and the world wide web, trends in Google searches by the public is a surrogate that can be used instead of time-consuming global or local surveys [2]. The global use of internet search engines has been increasing rapidly with an estimate of $46.8 \%$ of the global population accessing the internet in 2017, either due to personal choices or non-availability of time and resources for a timely hospital appointment (www.statista.com). The rate of such a cyberchondria or self-diagnosis by search engine is expected to rise to $53.7 \%$ by the year 2021 [3]. Recent data from internet live statistics showed a daily search rate of 3.5 billion searches on Google, equating to 1.2 trillion searches per year worldwide (www.internetlivestats.com).

Google trends is a platform provided by Google for researchers wherein it meticulously aides in quantifying the aggregates of millions of internet searches on a given topic and calculates its frequency offering results based on

Rajna Rasheed

rasheed.rajna@nhs.net

1 NIHR Moorfields Biomedical Research, Moorfields Eye Hospital, London EC1V 2PD, UK

2 Medical Retina, London, UK geographic locations and distinct time periods [4]. The Google trends tools scale the relative search results from 0 to 100 based on the most popular search term entered by the population at a particular region and time. It also helps to compare the relative search frequency of a maximum five distinct sets of search items at an attempt. Another important tool is a region-wise analysis of searches using Geo maps highlighting the different regions of a search area and search frequencies emerging from them. Numerous health specialties have resorted to use Google trends to understand the incidence and spread of diseases resulting in prediction of outbreaks in communicable diseases [5].

As diabetes is prevalent amongst the working age group and most people around the world have access to the World Wide Web, a survey of Google trends on global public awareness of diabetes, diabetic retinopathy or blindness was done. In addition, a comparison of the awareness of diabetic retinopathy to diabetic kidney disease and diabetic foot were performed. We assessed whether there was any change in search frequency during the period 2012 to 2019 compared with that in 2004-2011. This was analysed on a global scale and also for United Kingdom (UK) and India to demonstrate any regional differences. A region-wise analysis was done using Geo maps. In order to provide the scale of interest on diabetes compared with other interests, we studied India in more detail. We compared the Google trends in India for diabetic retinopathy or diabetes blindness relative to the Google trends on the Prime Minister of India, a famous Indian film actor and a famous cricketer and common eye conditions such as red eye and laser-assisted in-situ keratomileusis (LASIK) to compare the practise of utilizing such search tools in different regions of India.

The results are shown in Figs. 1 and 2. Google searches on diabetes and diabetic retinopathy have increased over time but are less frequent globally compared with the search for diabetes, diabetic kidney and diabetic foot. In the UK, these searches for diabetic retinopathy or diabetes-related blindness have declined over time compared with diabetic kidney disease and diabetic foot and may reflect the fact that UK already 
Google search trends for 'Diabetes'

Global

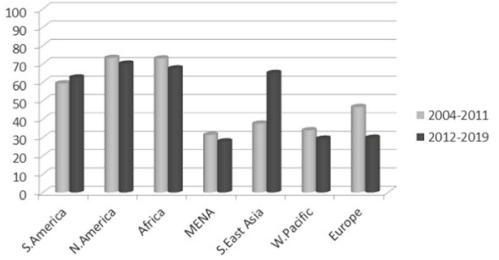

United Kingdom

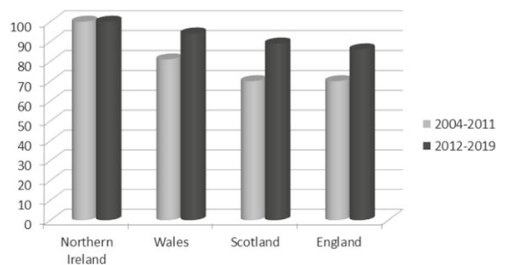

Ireteland
India

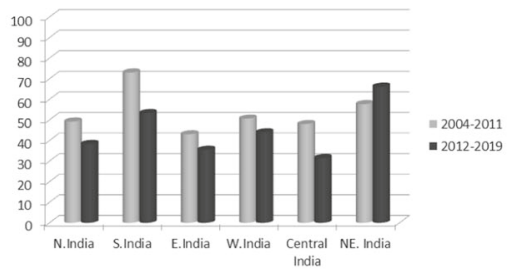

Google search trends for 'Diabetic Retinopathy or diabetes blindness'
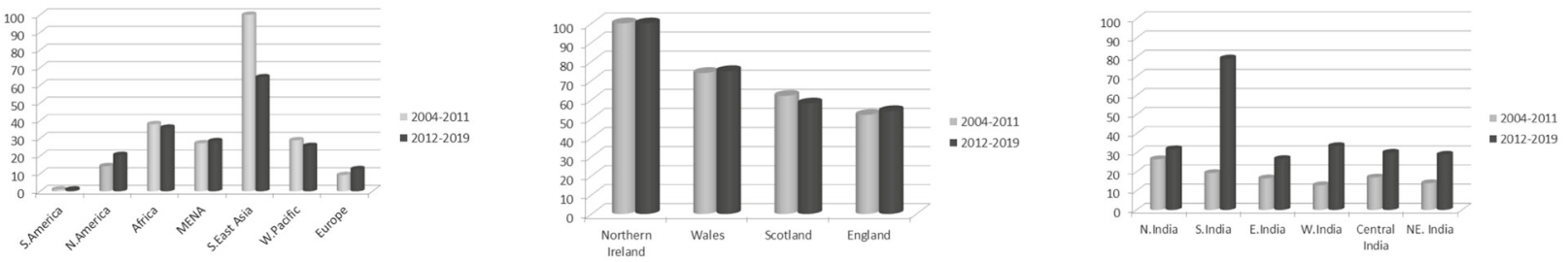

Google search trends for 'Diabetes Kidney'
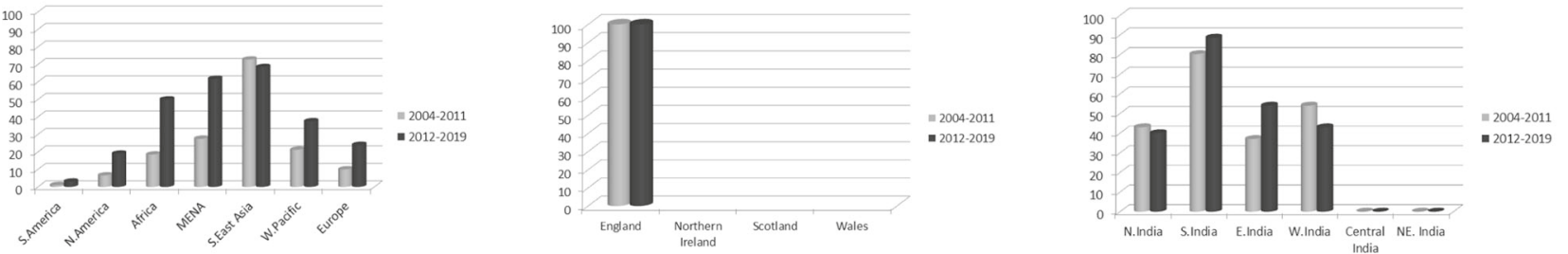

Google search trends for 'Diabetes Foot or diabetes foot ulcer'
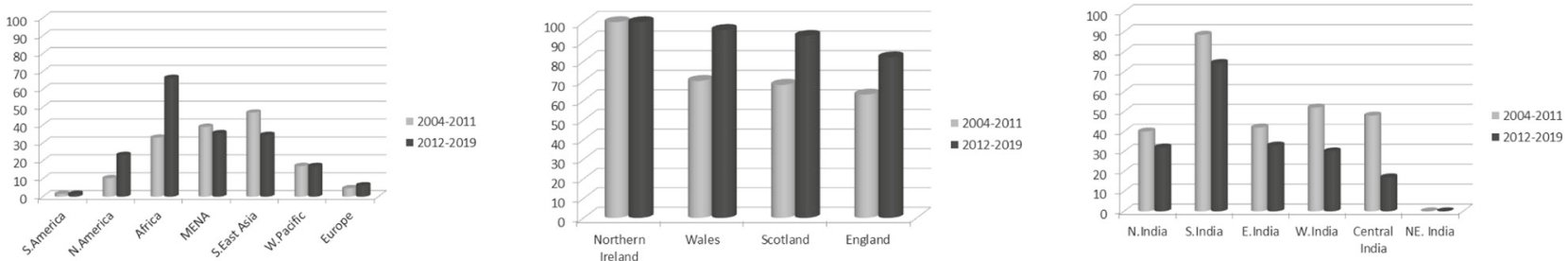

Fig. 1 Comparison of Google trends for diabetes and its complications. Graphs representing data from India, The United Kingdom and Overall Global statistics.

has a well-established diabetic retinopathy screening programme and so patients receive sufficient information from the care pathway. In contrast, in India, we observed that there is a higher inquisitiveness about diabetes and diabetes-related complications specifically diabetic retinopathy in South India compared with other parts of India and these search patterns have increased in last few years. These figures reflect the higher prevalence of diabetes in South India. The recent increase in searches for diabetic retinopathy is a positive sign of improving public awareness of the condition. The frequencies of searches on common eye conditions such as dry eye, red eye and LASIK show that the Indian public does resort to the 'Google Doctor' to gain knowledge on ocular conditions. Comparatively, the search intensity for diabetic retinopathy was less common. Unlike the UK, the public in
India is less likely to gain knowledge on diabetes retinopathy or blindness from the primary care system as it is still in its infancy. Therefore, these figures on relatively reduced searches for diabetic retinopathy and blindness depict the lack of awareness of this complication in the general population. The possibility of losing vision due to diabetes is yet to be emphasized among the Indian population. These study results show that directing people to informative diabetic retinopathy web sites using lay language may be a useful avenue of improving public awareness in countries where access to healthcare is challenging.

Another striking observation was that the Google searches for public figures were very high throughout India highlighting that these public figures have managed to draw immense public attention and could potentially act as 


\section{Google search trends for Indian \\ Prime Minister}

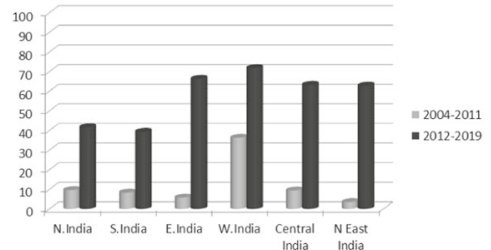

Google search trends for

Diabetic Retinopathy

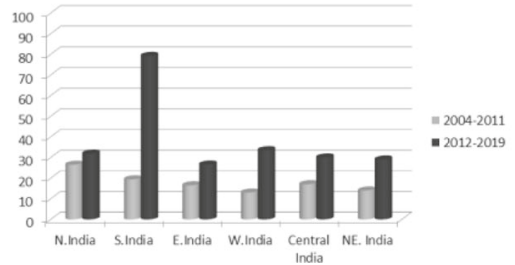

Google search trends for popular

Indian Celebrity (Film actor)

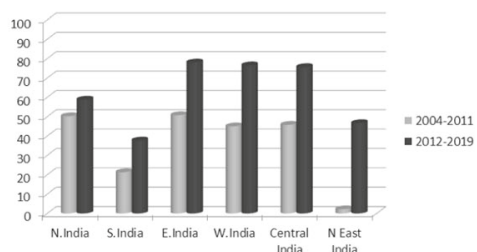

Google search trends for Dry Eye

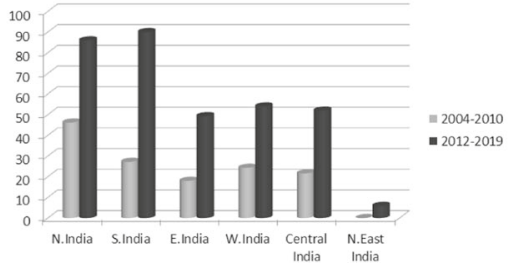

Google search trends for popular

Indian Celebrity (Cricketer)

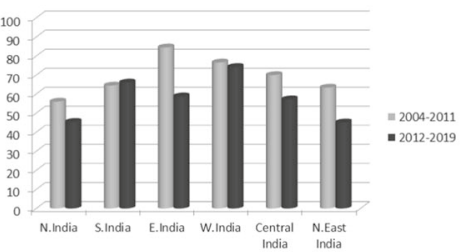

Google search trends for LASIK

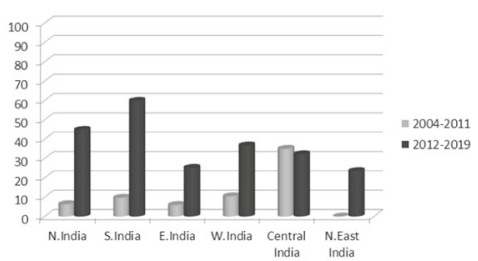

Fig. 2 Comparison of Google trends for diabetic retinopathy. Graphs depict comparison with other popular searches like public figures in India and other common eye diseases.

ambassadors to increase public awareness of diabetesrelated blindness, need for diabetic retinopathy screening and the need for treatment of sight-threatening complications. Given the global diabetes epidemic, similar public figures around the world, specific for each region, may be useful resources to spread awareness of this public health burden more rapidly than the usual practices of face-to-face meetings and distribution of pamphlets.

This study only reviewed Google trends and further studies on other social media may be useful tools for future surveys on public awareness of various aspects of diabetes and its complications.

Acknowledgements This study, within the ORNATE India project, is funded by the GCRF UKRI (MR/P207881/1). The research was supported by the NIHR Biomedical Research Centre at Moorfields Eye Hospital NHS Foundation Trust and UCL Institute of Ophthalmology.

\section{Compliance with ethical standards}

Conflict of interest The authors declare that they have no conflict of interest.
Publisher's note Springer Nature remains neutral with regard to jurisdictional claims in published maps and institutional affiliations.

\section{References}

1. Saeedi P, Petersohn I, Salpea P, Malanda B, Karuranga S, Unwin N, et al. Global and regional diabetes prevalence estimates for 2019 and projections for 2030 and 2045: results from the International Diabetes Federation Diabetes Atlas, 9th edition. Diabetes Res Clin Pract. 2019;157:107843.

2. Jacobs W, Amuta AO, Jeon KC. Health information seeking in the digital age: an analysis of health information seeking behavior among US adults. Cogent Soc Sci. 2017;3:130278.

3. Tkachenko N, Chotvijit S, Gupta N, Bradley E, Gilks C, Guo W, et al. Google trends can improve surveillance of Type 2 diabetes. Sci Rep. 2017;7:4993.

4. Deiner MS, Lietman TM, McLeod SD, Chodosh J, Porco TC. Surveillance tools emerging from search engines and social media data for determining eye disease patterns. JAMA Ophthalmol. 2016;134:102430.

5. Zhang Y, Bambrick H, Mengersen K, Tong S, Hu W. Using google trends and ambient temperature to predict seasonal influenza outbreaks. Environ Int. 2018;117:284-91. 